\title{
Farnesoid $X$ receptor agonist decreases lipid accumulation by promoting hepatic fatty acid oxidation in $\mathrm{db} / \mathrm{db}$ mice
}

\author{
YUJIE LIU $^{1 *}$, AN SONG ${ }^{2 *}$, XI YANG ${ }^{1}$, YUNFENG ZHEN $^{1}$, WEIWEI CHEN $^{1}$,

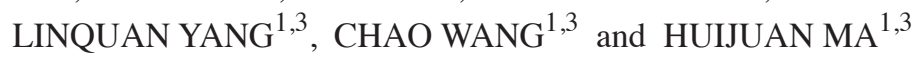 \\ ${ }^{1}$ Department of Endocrinology, Hebei General Hospital, Shijiazhuang, Hebei 050051; \\ ${ }^{2}$ Department of Endocrinology, Key Laboratory of Endocrinology, National Health and Family Planning Commission, \\ Peking Union Medical College Hospital, Chinese Academy of Medical Science and Peking Union Medical College, \\ Beijing 100191; ${ }^{3}$ Hebei Key Laboratory of Metabolic Disease, Shijiazhuang, Hebei 050051, P.R. China
}

Received November 26, 2017; Accepted May 30, 2018

DOI: $10.3892 / \mathrm{ijmm} .2018 .3715$

\begin{abstract}
The development of type-2 diabetes and its complications is associated with lipid metabolism disorder. Farnesoid $X$ receptor (FXR) has an important role in regulating lipid and glucose metabolism. However, the underlying mechanism of this remains unclear. The present study investigated the role of fexaramine (Fex), an FXR agonist, on lipid metabolism. For this purpose, 6-week-old $\mathrm{db} / \mathrm{db}$ mice were treated with Fex for 8 weeks via oral gavage and $\mathrm{db} / \mathrm{db}$ mice treated with corn oil were used as controls. Body weight and food intake were monitored daily and bi-weekly, respectively. A glucose tolerance test was performed during the final week of feeding. Blood samples were obtained for the analysis of lipids and enzymes related to hepatic function, and liver tissues were analyzed by histology and molecular examination. The results indicated that serum and liver triglyceride levels were decreased in $\mathrm{db} / \mathrm{db}$ mice administered with Fex. Fewer small lipid droplets were observed in the liver. Small heterodimer partner (SHP), a downstream gene of FXR, was upregulated following Fex treatment. The mRNA and protein expression of genes associated with fatty acid oxidation [acetyl coenzyme A carboxylase (ACC), carnitine palmitoyl transferase $1 \alpha(\mathrm{CPT} 1-\alpha)$ and peroxisome proliferator-activated receptor-coactivator-1 $\alpha$ ] was also increased. Additionally, the expression of AMP-activated protein kinase (AMPK) was also increased. However, the expression of sterol-regulatory element binding protein-1c and fatty acid synthase, which
\end{abstract}

Correspondence to: Dr Huijuan Ma, Department of Endocrinology, Hebei General Hospital, 348 He Ping West Road, Shijiazhuang, Hebei 050051, P.R. China

E-mail: huijuanma76@163.com

${ }^{*}$ Contributed equally

Key words: farnesoid $\mathrm{X}$ receptor, lipid accumulation, fatty acid oxidation, $\mathrm{db} / \mathrm{db}$ mice are associated with fatty acid synthesis, was not significantly different. Taken together, the results of the present study suggested that activation of FXR and its downstream gene SHP may induce the AMPK-ACC-CPT1- $\alpha$ signaling pathway, which promotes fatty acids oxidation, ultimately achieving its lipid-lowering effect.

\section{Introduction}

The prevalence of diabetes has been increasing in recent decades (1). Additionally, insulin resistance (IR) has been demonstrated to serve a crucial function in the development of type-2 diabetes mellitus (2). Fabbrini et al (3) recently reported that hepatic fatty acid metabolism disorder was associated with IR, and decreasing liver triglyceride (TG) accumulation in obese animals was associated with improved insulin sensitivity (4), strengthening the association between hepatic fatty acid metabolism and IR.

Farnesoid X receptor (FXR) is a member of the nuclear hormone receptor superfamily (5), which controls bile acid (BA) synthesis and transport, as well as lipid metabolism, through actions on the liver and intestines (6). In addition, Duran-Sandoval et al (7) using FXR knockout (KO) mice have identified a critical role of FXR in peripheral insulin signaling and hepatic glucose metabolism. FXR KO mice display glucose intolerance and insulin insensitivity; activation of FXR with the GW4064 agonist, or with a virus overexpressing a constitutively active form of FXR in the liver; lowered blood glucose levels in both $\mathrm{db} / \mathrm{db}$ and wild-type mice by repressing gluconeogenic genes in the liver; and activating hepatic glycogen synthesis $(8,9)$. However, the underlying mechanisms through which FXR alters hepatic fatty acid metabolism remain undetermined.

Fexaramine (Fex) is a selective agonist of FXR. It is a non-BA synthetic activator with marked selectivity for FXR over other BA receptors, including pregnane $\mathrm{X}$ receptor, constitutive androstane receptor, and vitamin $\mathrm{D}$ receptor (10). In the present study, db/db mice were treated with Fex, in order to evaluate the regulatory effects of activation of FXR on hepatic glucose and fatty acid metabolism, and to elucidate the mechanisms of this. 


\section{Materials and methods}

Animal models. Male $\mathrm{db} / \mathrm{db}$ mice ( $\mathrm{n}=20$; age, 5 weeks; weight, 18-23 g) were purchased from the Nanjing Biomedical Research Institute of Nanjing University (Nanjing, China). Mice ( $\mathrm{n}=5 /$ cage) were maintained at a constant temperature $\left(23 \pm 2^{\circ} \mathrm{C}\right)$ and humidity $(50 \pm 10 \%)$ in a 12 -h light/dark cycle. Mice had ad libitum access to standard chow (consisting of $10 \%$ fat, $70 \%$ carbohydrate and $20 \%$ protein) and water. Following one week of acclimatization, mice were randomly divided into two experimental groups (n=10/group): Fex group and control (Con) group. The Fex group was administered $50 \mathrm{mg} / \mathrm{kg}$ body weight Fex [in corn oil, as detailed by Fang et al (11); MedchemExpress, Monmouth Junction, NJ, USA] by gavage daily for 8 weeks, whereas the control group received corn oil. A glucose tolerance test (GTT) was performed during the final week of feeding as described below. All experiments involving animals were performed according to the procedures approved by the Institutional Animal Care and Use Committee of the Institute of Zoology, Hebei General Hospital (Shijiazhuang, China). The mice were euthanized via cervical dislocation.

Biological assays. The body weight of each mouse was recorded every day; the quantity of food consumed by each cage was recorded twice a week, and the mean food consumption per mouse was calculated. Following the experimental period, the animals were fasted overnight and sacrificed. Liver tissue was surgically removed from each mouse, weighed, snap frozen in liquid nitrogen, and stored at $-80^{\circ} \mathrm{C}$ for future analysis. Blood samples were collected from the orbital vein for the measurement of serum indexes. Blood glucose levels were measured using an Accuchek Active Meter (Roche Applied Science, Penzberg, Germany) according to the manufacturer's instructions. Enzyme kits were used to measure the total cholesterol (TC; A110-1), TG (A111-1), free fatty acid (FFA; A042-2; all from Nanjing Jiancheng Bioengineering Institute, Nanjing, China), aspartate aminotransferase (AST) and alanine aminotransferase (ALT) (both from Sigma-Aldrich; Merck KGaA, Darmstadt, Germany).

Intraperitoneal (i.p.) GTT and serum insulin. Following 8 weeks of treatment, mice were subjected to an i.p. GTT. Mice received i.p. injection of $2 \mathrm{~g} / \mathrm{kg}$ glucose following $12 \mathrm{~h}$ fasting. Glucose levels were measured from blood collected from the tail using an Accuchek Active Meter immediately prior to and 15, 30, 60 and 120 min following i.p. glucose injection. For insulin, serum was prepared from blood by centrifugation (at $2,000 \mathrm{xg}$ for $10 \mathrm{~min}$ at $37^{\circ} \mathrm{C}$ ), and measured with insulin ELISA kits (ALPCO Diagnostics, Salem, NH, USA), according to the manufacturer's guidelines.

Assay of TG in liver tissue. Liver TG content was measured as follows: Liver samples were weighed and homogenized with anhydrous ethanol $(1 \mathrm{~g} ; 9 \mathrm{ml})$ and centrifuged at 1,400 x g for $10 \mathrm{~min}$ at $4^{\circ} \mathrm{C}$. The supernatant was collected to determine the total amount of tissue lipids, using the ELISA kit according to the manufacturer's protocol (Nanjing Jiancheng Bioengineering Institute).
Hematoxylin and eosin $(H \& E)$ staining and Oil-Red-O staining. Liver samples were collected from mice following 8 weeks of treatment. Samples were frozen and cut into $5-\mathrm{mm}$ sections. The sliced sections were treated with H\&E and also stained with Oil Red O using a light micoscope as previously described (12).

Reverse transcription-quantitative polymerase chain reaction $(R T-q P C R)$. Total RNA was isolated from liver tissue using TRIzol (Invitrogen; Thermo Fisher Scientific, Inc., Waltham, MA, USA). The mRNA concentration was quantified using a Nano Drop spectrophotometer (Thermo Fisher Scientific, Inc.). The diluted mRNA $(0.5 \mathrm{mg} / \mathrm{ml})$ was reverse-transcribed using the Total RevertAid First Strand cDNA synthesis kit (Thermo Fisher Scientific, Inc.) according to the manufacturer's protocol, and the gene expression levels were determined on ABI 7300 Real-Time PCR System (Applied Biosystems, USA) using the Syber Green I Go Taqs Qpcr Detection kit (Tiangen Biotech Co., Ltd., Bejing, China) and normalized to GAPDH expression using the $2^{-\Delta \Delta C q}$ method (13). The standard cycling conditions were as follows: $95^{\circ} \mathrm{C}$ for $3 \mathrm{~min}$, followed by 40 cycles at $95^{\circ} \mathrm{C}$ for $5 \mathrm{sec}, 60^{\circ} \mathrm{C}$ for $10 \mathrm{sec}$ and $72^{\circ} \mathrm{C}$ for $15 \mathrm{sec}$. The primer sequences for each gene are listed in Table I.

Western blotting. The nuclear, cytosolic and membrane fractions were extracted from liver tissue as previously described (14). To obtain total proteins, liver tissues from the mice were lysed in a buffer containing $50 \mathrm{mM}$ Tris- $\mathrm{HCl}$ (pH 7.4), $150 \mathrm{mM} \mathrm{NaCl}, 1 \%$ Triton X-100, $0.1 \%$ SDS and $1 \mathrm{mM}$ phenylmethylsulfonyl fluoride. The protein concentration was measured using a bicinchoninic acid assay method (Beijing Solarbio Science and Technology, Beijing, China). Lysates of $10-15 \mu \mathrm{g}$ protein were separated by $10 \%$ SDS-PAGE gel, transferred onto polyvinylidene difluoride membranes (EMD Millipore, Billerica, MA, USA), blocked with 5\% skimmed dry milk at room temperature for $1 \mathrm{~h}$, and probed with primary antibodies at $4^{\circ} \mathrm{C}$ overnight. Antibodies against FXR (ab129089, anti-rabbit; 1:1,000), small heterodimer partner (SHP; ab32559, anti-rabbit; 1:1,000), AMP-activated protein kinase (AMPK; ab32047, anti-rabbit; 1:1,000), phosphorylated (p)-AMPK (ab133448, anti-rabbit; 1:1,000), acetyl coenzyme A carboxylase (ACC; ab45174, anti-rabbit; 1:2,000), p-ACC (ab169768, anti-rabbit; 1:1,000), carnitine palmitoyl transferase $1 \alpha(\mathrm{CPT} 1-\alpha$; ab128568, anti-mouse; 1:800), peroxisome proliferator-activated receptor-coactivator- $1 \alpha$ (PGC- $1 \alpha$; ab54481, anti-rabbit; 1:1,000), sterol-regulatory element binding protein (SREBP)-1c (ab28481, anti-rabbit; 1:800) and fatty acid synthase (Fas; ab82419, anti-rabbit; 1:1,000) were all purchased from Abcam (Cambridge, UK). The blots were subsequently incubated with horseradish peroxidase-conjugated secondary antibodies, the secondary antibodies used were peroxidase-conjugated rabbit (sc2031) or mouse (sc2032) antibodies (1:10,000; both from GE Healthcare Bio-Sciences, Pittsburgh, PA, USA) followed by detection via enhanced chemiluminescence (sc2048; Santa Cruz Biotechnology, Inc., Dallas, TX, USA). The protein bands were quantified by densitometry using ImageJ software (version 1.51k; National Institutes of Health, Bethesda, MD, USA). Normalization was performed by blotting the same samples with an antibody against GAPDH (ab54481, anti-rabbit; 1:10,000; Abcam). 

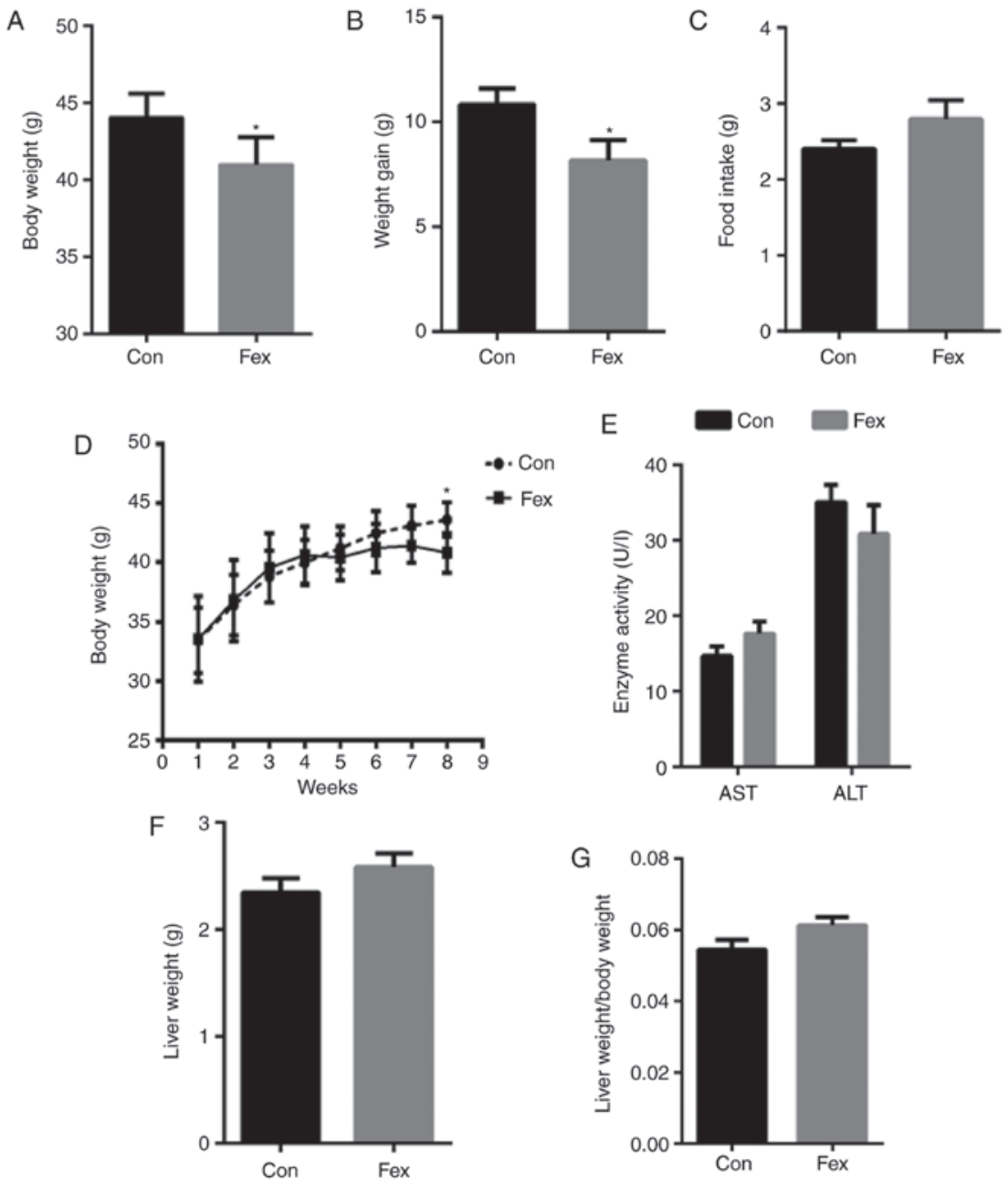

Figure 1. Comparison of (A) body weight, (B) weight gain, (C) food intake, (D) body weight growth (from the beginning of the intervention to the end), (E) AST and ALT, (F) liver weight, and (G) the ratio of liver weight to body weight between the Con and Fex groups following 8 weeks of treatment. Data are presented as the mean + or \pm standard error of the mean, $(\mathrm{n}=10)$. ${ }^{*} \mathrm{P}<0.05$ vs. Con group. AST, aspartate aminotransferase; ALT, alanine aminotransferase; Con, control; Fex, fexaramine.

Statistical analysis. All data are presented as the mean \pm standard error of the mean. Student's t-test was performed for comparisons between two groups. SPSS version 21.0 software (IBM Corp., Armonk, NY, USA) was used for the statistical analysis. $\mathrm{P}<0.05$ was considered to indicate a statistically significant difference.

\section{Results}

Body weight and weight gain decreases significantly with fexaramine treatment in $d b / d b$ mice. Following 8 weeks of treatment, mice in the Fex group exhibited a significant decrease in body weight and weight gain compared with the Con group, whereas there was no significant difference in mean food intake per day between the two groups (Fig. 1A-D). In order to exclude the idea that the lower body weight did not result from the pathological effects of Fex, ALT and AST levels in the serum were also detected, and the results indicated that there was no statistically significant difference in ALT and AST levels between the Con and Fex groups (Fig. 1E). This indicated that the reduction in body weight was a result of Fex and was independent of food intake and the pathological effect of Fex on the liver.
To further investigate the effects of Fex on liver weight, liver weight was also recorded. There were no significant differences in the liver weight or the liver/body weight ratio between the Fex group and the Con group at the end of the experiment (Fig. 1F and G).

Effects of Fex on blood glucose, serum insulin and insulin sensitivity. To determine the effects of Fex on blood glucose and insulin, fasting blood glucose and serum insulin were measured, and the results indicated that both fasting blood glucose and serum insulin were not significantly different between the two groups (Fig. 2A and B); the authors speculate that this was likely due to the short intervention time of Fex. To test whether Fex improved insulin sensitivity, an i.p. GTT was performed following 8 weeks of Fex treatment. I.p. GTT indicated significantly reduced blood glucose levels following glucose loading at $15 \mathrm{~min}$ in the Fex group, whereas glucose levels at $0,30,60$ and $120 \mathrm{~min}$ did not significantly differ between the two groups (Fig. 2C). The area under the curve (AUC) was then calculated. In accordance with the above results, the AUC of the Fex group was markedly lower than that of the Con group (Fig. 2D), thus suggesting improved insulin sensitivity with Fex treatment. 

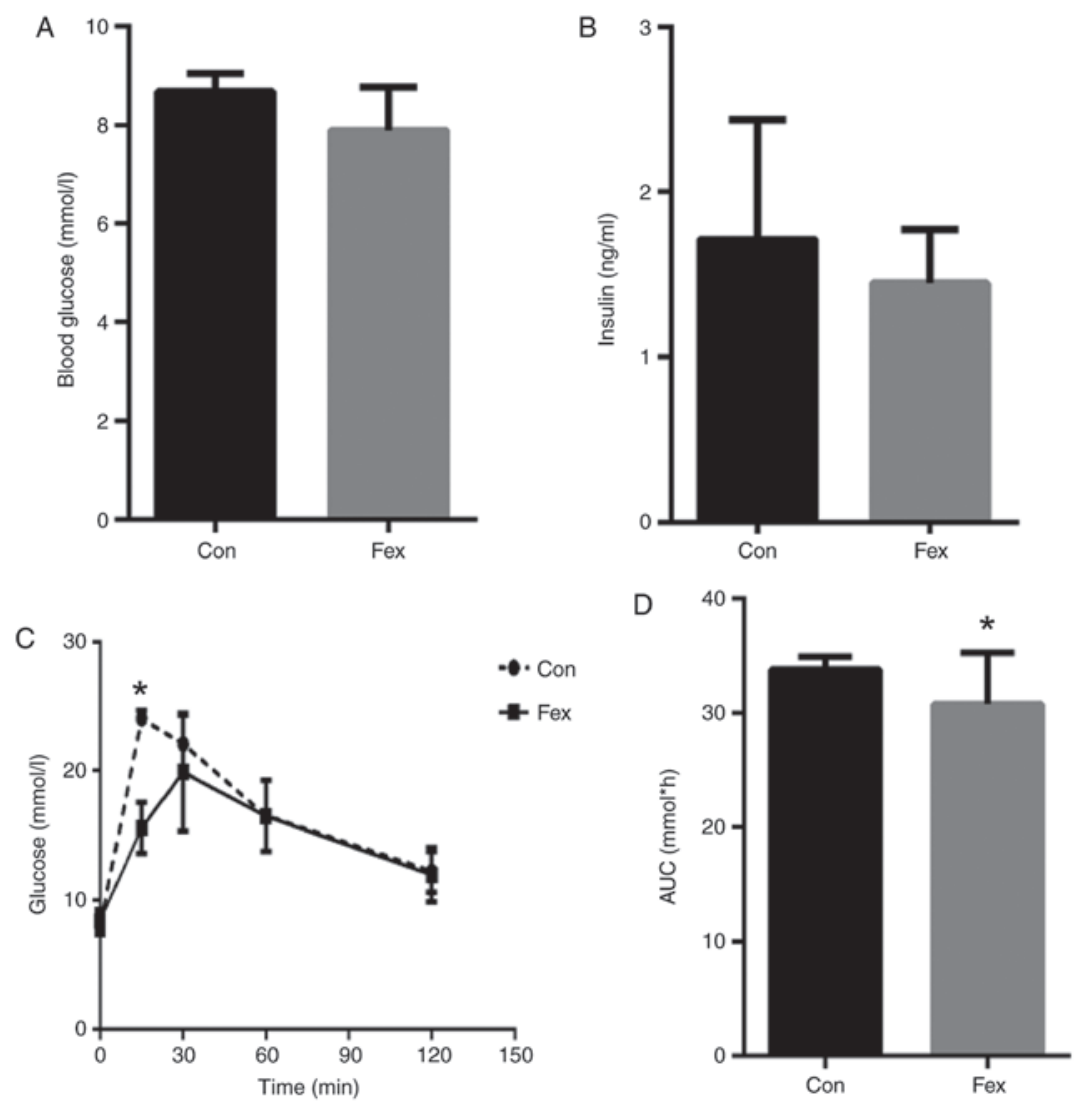

Figure 2. (A) Blood glucose and (B) insulin level at the end of intervention. (C) An intraperitoneal glucose tolerance test was performed following 8 weeks of treatment and (D) the AUC was calculated. Data are presented as the mean + standard error of the mean, $(\mathrm{n}=10)$. " $\mathrm{P}<0.05$ vs. Con group. AUC, area under the curve; Con, control; Fex, fexaramine.

Table I. Primers used for gene expression analysis.

\begin{tabular}{lll}
\hline Gene & \multicolumn{1}{c}{ Forward primer sequence (5'-3') } & \multicolumn{1}{c}{ Reverse primer sequence (5'-3') } \\
\hline GAPDH & ACCCAGAAGACTGTGGATGG & GGAGACAACCTGGTCCTCAG \\
FXR & GCCATCAAGGACGTCAGCA & CTTCCTCCGAGTAGCGAATCAG \\
SHP & CGATCCTCTTCAACCCAGATG & AGGGCTCCAAGACTTCACACA \\
AMPK & ATCCAAACACCAAGGCGT & TTCCATTCATAGTCCAACTGCT \\
CPT1- $\alpha$ & CGCACGGAAGGAAAATG & TGTGCCCAATATTCCTGG \\
PGC- $1 \alpha$ & ACCAAACCCACAGAGAACAG & GGGTCAGAGGAAGAGATAAAGTTG \\
ACC & TGACAGACTGATCGCAGAGAAAG & TGGAGAGCCCCACACACA \\
SREBP-1c & GGAGCCATGGATTGCACATT & GCTTCCAGAGAGGAGGCCAG \\
Fas & GCTGCGGAAACTTCAGGAAAT & AGAGACGTGTCACTCCTGGACTT
\end{tabular}

FXR, farnesoid X receptor; SHP, small heterodimer partner; AMPK, AMP-activated protein kinase; CPT1- $\alpha$, carnitine palmitoyl transferase $1 \alpha$; PGC-1 $\alpha$, peroxisome proliferator-activated receptor-coactivator- $1 \alpha$; ACC, acetyl coenzyme A carboxylase; SREBP-1c, sterol-regulatory element binding protein-1c; Fas, fatty acid synthase.

Improved liver and serum lipid profile in Fex-treated mice. Following 8 weeks of treatment with Fex, serum and liver TG levels were significantly decreased in the Fex group compared with the Con group (Fig. 3A and B). Serum TC in the Fex group exhibited no significant difference compared with the Con group (Fig. 3C). Serum FFA was significantly decreased in the Fex group, compared with controls (Fig. 3D). As revealed by $H \& E$ staining, the number and size of lipid droplets in the livers of the mice treated with Fex were also markedly reduced (Fig. 3E and F).

Effect of Fex on genes associated with hepatic fatty acid metabolism. RT-qPCR and western blotting were used to measure the mRNA and protein expression level, respectively, of certain genes associated with fatty acid metabolism in the livers of mice treated with or without Fex. Both mRNA and 

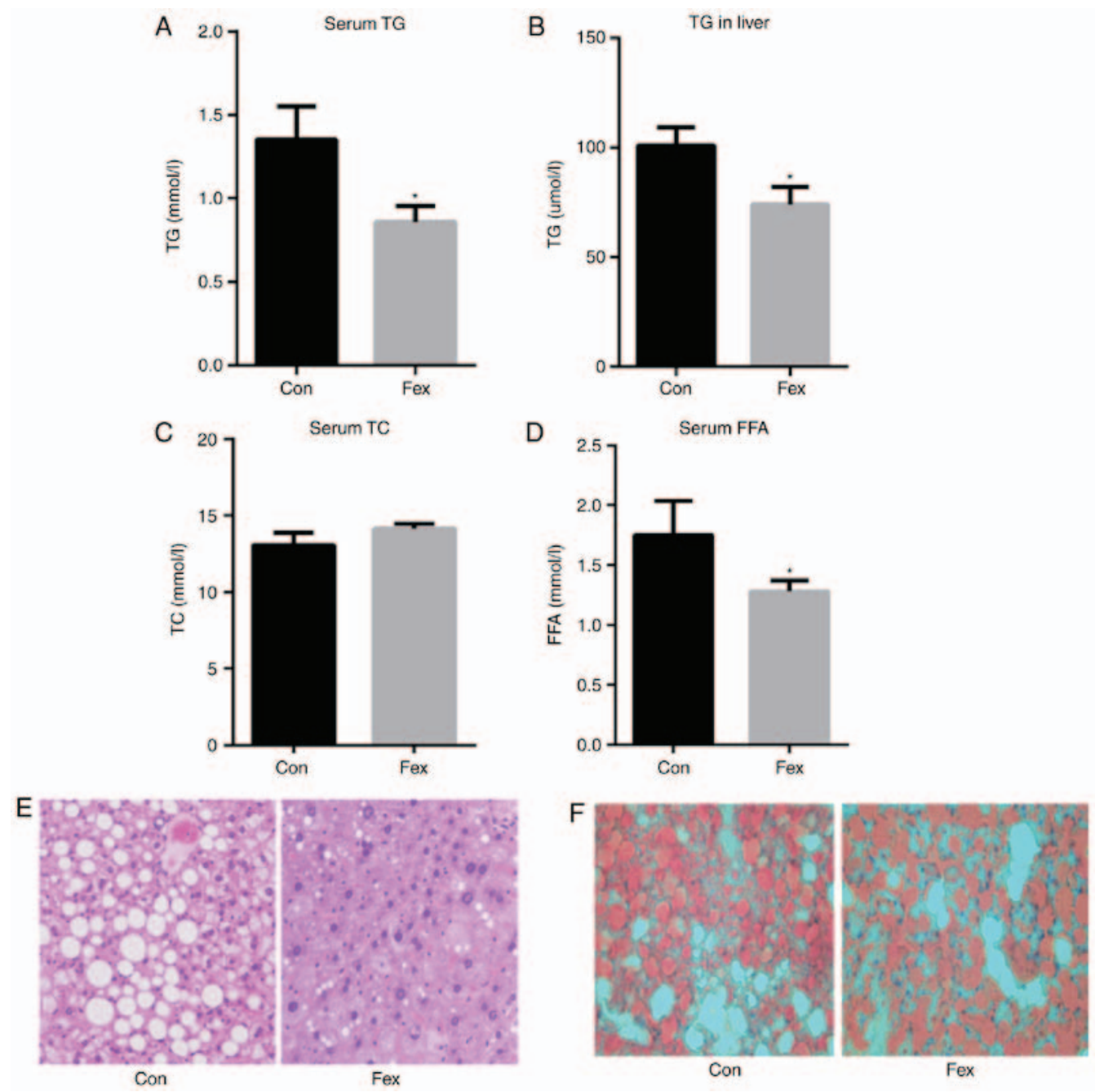

Figure 3. Effect of Fex on (A) serum TG, (B) hepatic TG, and serum (C) TC and (D) FFA following 8 weeks of treatment. Effect of Fex on liver lipid generation assessed via (E) hematoxylin and eosin, and (F) Oil-Red-O staining (original magnification, $\mathrm{x} 400$ ). Data are presented as the mean + standard error of the mean, ( $\mathrm{n}=10$ ). "P<0.05 vs. Con group. Fex, fexaramine; TG, triglycerides; TC, total cholesterol; FFA, free fatty acids; Con, control.
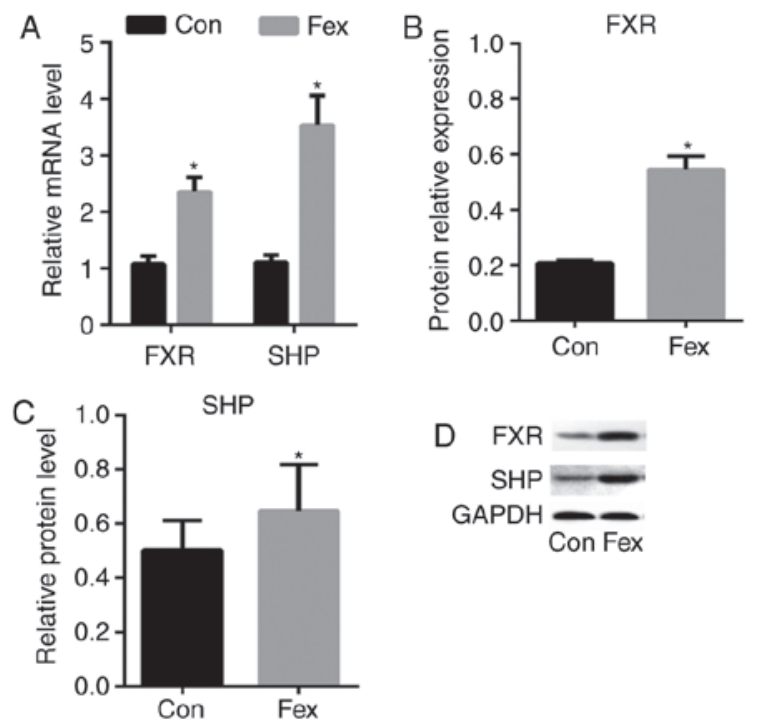

Figure 4. (A) The mRNA expression levels of FXR and SHP in the liver. The protein expression levels of (B) FXR and (C) SHP as calculated via densitometric analysis of (D) western blotting. Data are presented as the mean + standard error of the mean, $(n=5) .{ }^{*} \mathrm{P}<0.05$ vs. Con group. FXR, farnesoid X receptor; SHP, small heterodimer partner; Con, control; Fex, fexaramine. protein expression levels of FXR were significantly higher in the livers of the Fex group than in the Con group, and SHP, a downstream gene of FXR exhibited a similar change (Fig. 4), which indicated that Fex can activate hepatic FXR signaling. Lipogenesis and fatty acid oxidation are two key metabolic pathways that regulate hepatic triacylglycerol metabolism (15). The protein and mRNA expression levels of the genes associated with these two pathways were evaluated. The mRNA expression levels of AMPK, CPT1- $\alpha$ and PGC- $1 \alpha$ and protein expression of p-AMPK/AMPK, p-ACC, CPT1- $\alpha$ and PGC- $1 \alpha$, which are associated with fatty acid oxidation, were significantly increased by Fex treatment, whereas the total ACC did not markedly differ between the two groups (Fig. 5). SREBP-1c and Fas, which were associated with lipogenesis, did not significantly differ between the two groups both in mRNA and protein levels (Fig. 6). These results indicated that hepatic FXR signaling can reduce serum lipid, probably by activating fatty acid oxidation.

\section{Discussion}

A previous study demonstrated that Fex could protect against diet-induced weight gain (11). Ryan et al (6) had demonstrated 


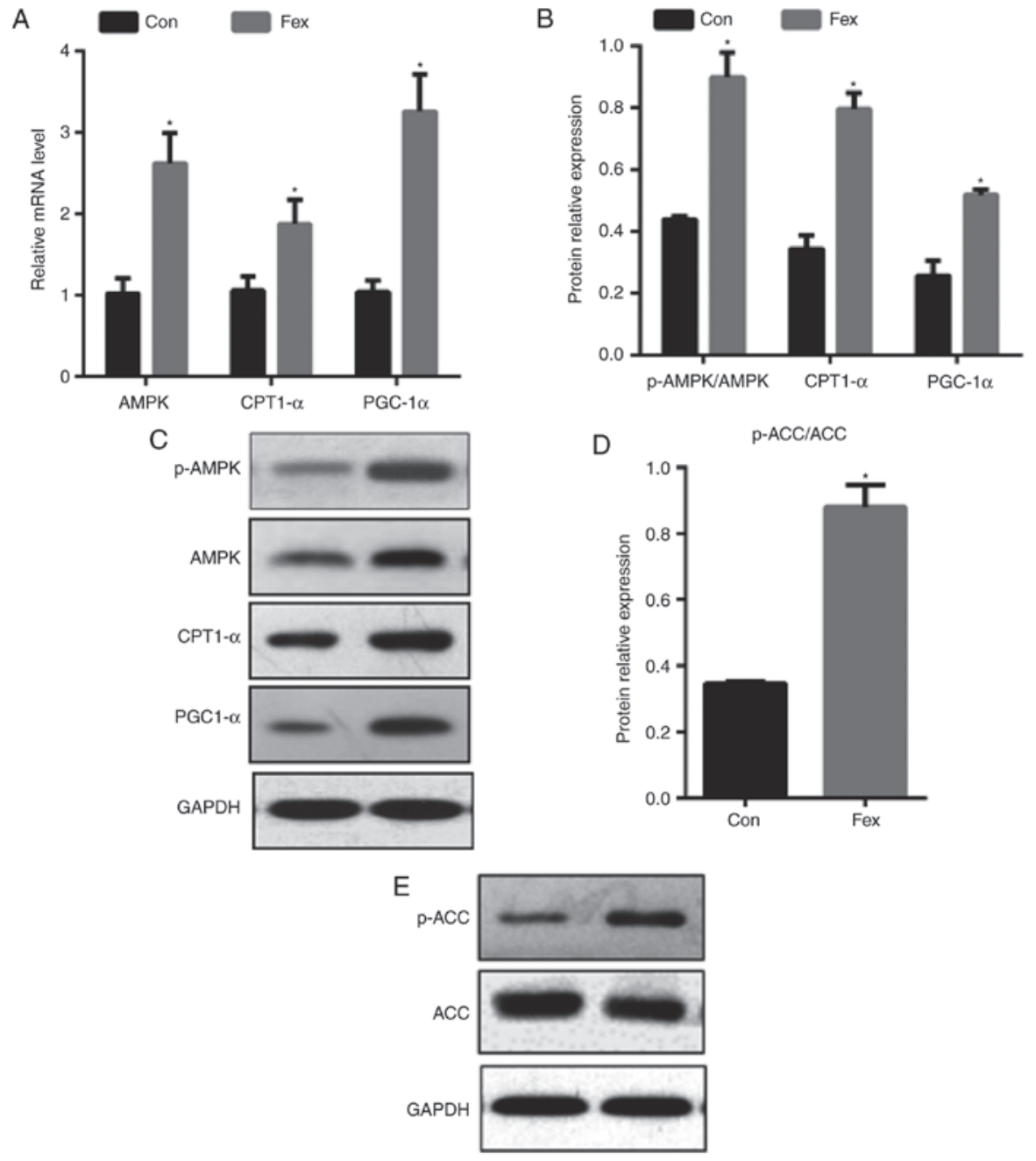

Figure 5. Effect of Fex on fatty acid oxidation in the liver. (A) The mRNA expression levels of AMPK, CPT1- $\alpha$ and PGC-1 $\alpha$. (B-E) The protein expression levels of p-AMPK/AMPK, p-ACC/ACC, CPT1- $\alpha$ and PGC-1 $\alpha$ were examined by western blotting and densitometric analysis of protein expression. Data are presented as the mean + standard error of the mean, $(n=5)$. ${ }^{*} \mathrm{P}<0.05$ vs. Con group. Fex, fexaramine; AMPK, AMP-activated protein kinase; ACC, acetyl coenzyme A carboxylase; CPT1- $\alpha$, carnitine palmitoyl transferase $1 \alpha$; PGC-1 $\alpha$, peroxisome proliferator-activated receptor-coactivator-1 $\alpha$; p-, phosphorylated; Con, control.
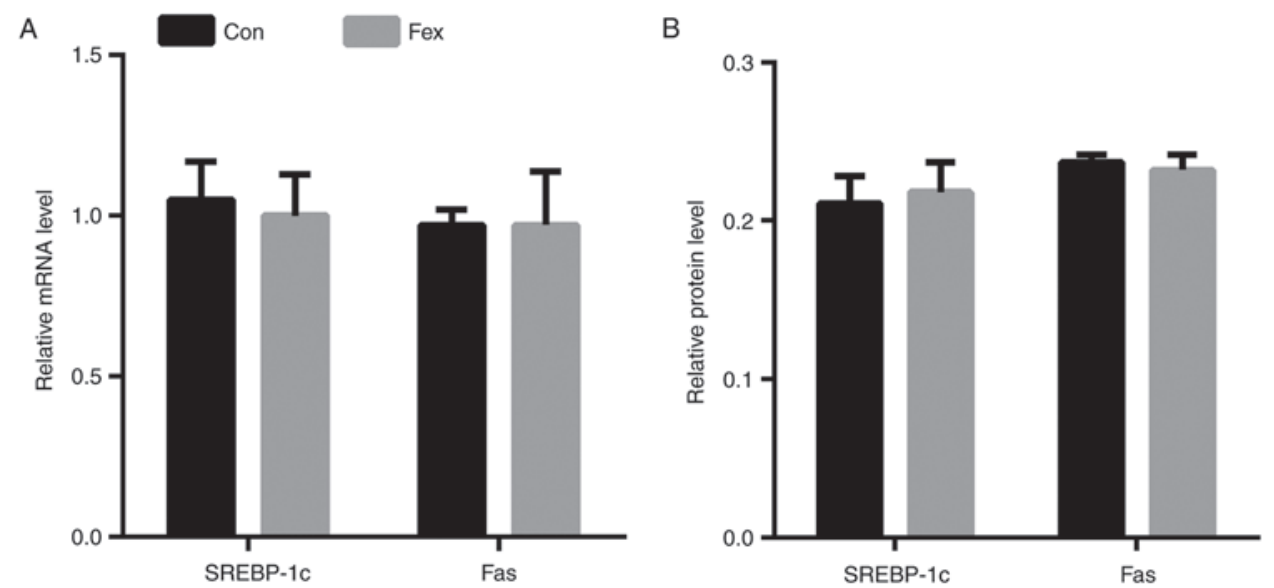

C

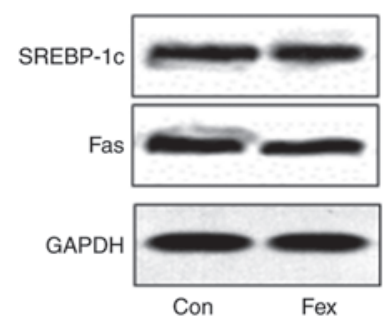

Figure 6. Effect of Fex on lipogenesis in the liver. (A) The mRNA expression levels of SREBP-1c and Fas. (B and C) The protein expression levels of SREBP-1c and Fas were examined by western blotting and densitometric analysis of protein expression. Data are presented as the mean + standard error of the mean, $(n=5)$. Fex, fexaramine; SREBP-1c, sterol-regulatory element binding protein-1c; Fas, fatty acid synthase; Con, control.

that the impact of vertical sleeve gastrectomy on body weight and glucose tolerance appeared lesser in FXR deficient mice, suggesting an association between FXR and body weight. In the present study, it was demonstrated that mice fed with Fex 
exhibited a significant decrease in body weight and weight gain compared with the Con group, and the change was independent of food intake and the pathological effect of Fex on the liver.

Hepatic steatosis is closely associated with liver weight and de Oliveira et al (16) previously demonstrated that a high-fat diet can result in significant body weight gain in C57BL mice. The current study revealed that Fex intervention for 8 weeks could significantly reduce body weight compared with the animals of the model group. However, there was no significant difference in the liver weight and liver/body weight ratio between the Fex group and the Con group animals in the present study. The $\mathrm{db} / \mathrm{db}$ mouse is a diabetes animal model associated with leptin deficiency, and does not require a high-fat diet (17); therefore, the liver weight and steatosis is not very clear compared with the high-fat diet-induced model. In the present study, $\mathrm{db} / \mathrm{db}$ mice were used as a diabetes animal model, which may be the main reason why liver weight was not changed by Fex intervention. Another reason for this result may be the short duration of the drug intervention; it may be speculated that a long term Fex treatment for 12 weeks might induce more notable changes in $\mathrm{db} / \mathrm{db}$ mice.

In diabetic animals, FXR expression is diminished, suggesting a link between FXR and glucose metabolism (18). Accordingly, it has been demonstrated that FXR activation in $\mathrm{db} / \mathrm{db}$ mice could alleviate hyperglycemia, enhancing insulin response (8). Consistently, mice with FXR deficiency displayed peripheral insulin resistance and defects in their glucose disposal rate (19). In the current study, Fex was used, an FXR-specific agonist to activate FXR in the liver, and demonstrated that activation of FXR could result in both hypoglycemia and an improvement in insulin sensitivity. The effects of Fex treatment, such as hypoglycemia and improved insulin sensitivity, can be illustrated by the decreased blood glucose levels following glucose loading in $15 \mathrm{~min}$, and the AUC in the Fex group. However, fasting blood glucose and serum insulin did not differ significantly between the two groups; therefore, the reason for this phenomenon requires further study to be elucidated.

Lambert et al (20) used FXR KO mice to verify the functional role of FXR. They demonstrated that FXR KO mice exhibited higher serum levels of BA, TG and FFAs in close association with increased high-density lipoprotein cholesterol and lipoprotein lipase activity. Furthermore, another study demonstrated that FXR KO mice are characterized by elevated plasma bile salt, TG and cholesterol levels, as well as increased hepatic TG and cholesterol levels (21). These findings all demonstrated that there is a close association between FXR and lipid metabolism. In the present study, it was demonstrated that TG levels in the serum and liver of mice gavaged with Fex were significantly decreased, and lipid droplets in the liver also appeared to be reduced in both number and size. Based on these findings, it was speculated that activation of FXR exerted an effect of lowering TG levels in both the plasma and the liver.

AMPK has a central role in controlling lipid metabolism through modulating the downstream ACC and carnitine palmitoyl transferase 1 (CPT1) pathway (22). The phosphorylation of ACC at Ser79 by AMPK leads to the inactivation of ACC (23), and the level of Ser79 phosphorylation is commonly used as an in-vivo measure of AMPK signaling in response to various stimuli (24). CPT1 catalyzes the first rate-limiting step in the $\beta$ oxidation of long-chain fatty acids in mitochondria and it can be inhibited by unphosphorylated ACC (25). There are three isoforms of CPT1: CPT1 $\alpha$, CPT1 $\beta$ and CPT1c. Of the three isoforms of CPT1, only CPT1 $\alpha$ localizes in the mitochondria (26). AMPK extracellular signaling is the regulated kinase pathway downstream of FXR (27). AMPK activity was determined by measuring the phosphorylation level of the AMPKa subunit at Thr172, which reflects the activation of AMPK (28). A previous study also demonstrated that FXR can upregulate the expression of SHP (29). In the current study, it was demonstrated that, upon FXR activation, SHP expression was upregulated, and the expression of p-AMPK/AMPK, ACC and CPT1 $\alpha$ was increased, which induces fatty acid $\beta$ oxidation, thereby improving lipid metabolism. Based on the aforementioned results, it was hypothesized that FXR may induce fatty acid $\beta$ oxidation through the AMPK-ACC-CPT1 $\alpha$ pathway.

PGC- $1 \alpha$ is a transcription co-factor that interacts with numerous transcription factors, and has been demonstrated to be a potent activator of mitochondrial biogenesis and fatty acid oxidation (30). Hepatic PGC-1 $\alpha$ protein expression and transcriptional activation of mitochondrial biogenesis have been reported to be lower in a rodent model with hepatic steatosis (31). Furthermore, an elevation in hepatic PGC-1 $\alpha$ expression results in increased mitochondrial content and/or function, increased complete fatty acid oxidation and TCA cycle flux, and reduced hepatic triacylglycerol content and secretion (32). Therefore, it was further investigated whether PGC-1 $\alpha$ mediates the lipid-lowering effect of FXR. The results demonstrated that PGC-1 $\alpha$ was significantly increased by Fex treatment, which indicated that FXR may increase the expression of PGC-1 $\alpha$ in the liver to exert a lipid-lowering effect.

Previous studies have reported that activation of FXR lowers plasma triglyceride levels by a mechanism that involves the repression of hepatic SREBP-1c expression $(33,34)$. However, Matsukuma et al (35) reported that although activation of FXR represses SREBP-1c expression, activation of FXR does not suppress SREBP-1c target genes, including fatty acid synthase. In the current study, it was demonstrated that the relative protein expression of both SREBP-1c and Fas did not significantly differ between the Fex group and the Con group, and the inconsistencies of these studies require further investigation.

In conclusion, the present study indicated that $\mathrm{db} / \mathrm{db}$ mice treated with Fex exhibited lower TG and FFA levels and improved lipid metabolism, and this may be achieved via sequential events involving the activation of FXR by Fex, and activation of the FXR downstream signaling pathway, AMPK-ACC-CPT1 $\alpha$, which results in increase of fatty acid $\beta$ oxidation, ultimately achieving lipid-lowering effects. These findings may provide a mechanistic basis for targeting FXR as a potential therapeutic strategy to combat dyslipidemia in diabetic patients.

\section{Acknowledgements}

Not applicable. 


\section{Funding}

The present study was funded by the International Cooperation Program of Hebei Province (grant no. 15397750D).

\section{Availability of data and materials}

The datasets used and analyzed during the current study are available from the corresponding author on reasonable request.

\section{Authors' contributions}

YL collected and analyzed the majority of the data and wrote the manuscript. HM collected and analyzed data and edited the manuscript. AS, XY, YZ, WC and LY collected data. CW provided input regarding data analysis and statistics. HM was involved in study design, oversaw data collection and analysis, and wrote and edited the manuscript.

\section{Ethics approval and consent to participate}

All experiments involving animals were performed according to the procedures approved by the Institutional Animal Care and Use Committee of the Institute of Zoology, Hebei General Hospital (Shijiazhuang, China).

\section{Patient consent for publication}

Not applicable.

\section{Competing interests}

The authors declare that they have no competing interests.

\section{References}

1. Guariguata L, Whiting DR, Hambleton I, Beagley J, Linnenkamp U and Shaw JE: Global estimates of diabetes prevalence for 2013 and projections for 2035. Diabetes Res Clin Pract 103: 137-149, 2014.

2. Samuel VT, Petersen KF and Shulman GI: Lipid-induced insulin resistance: Unravelling the mechanism. Lancet 375: 2267-2277, 2010.

3. Fabbrini E and Magkos F: Hepatic steatosis as a marker of metabolic dysfunction. Nutrients 7: 4995-5019, 2015.

4. Monsénégo J, Mansouri A, Akkaoui M, Lenoir V, Esnous C, Fauveau V, Tavernier V, Girard J and Prip-Buus C: Enhancing liver mitochondrial fatty acid oxidation capacity in obese mice improves insulin sensitivity independently of hepatic steatosis. J Hepatol 56: 632-639, 2012.

5. Forman BM, Goode E, Chen J, Oro AE, Bradley DJ, Perlmann T, Noonan DJ, Burka LT, McMorris T, Lamph WW, et al Identification of a nuclear receptor that is activated by farnesol metabolites. Cell 81: 687-693, 1995.

6. Ryan KK, Tremaroli V, Clemmensen C, Kovatcheva-Datchary P, Myronovych A, Karns R, Wilson-Pérez HE, Sandoval DA, Kohli R, Bäckhed F and Seeley RJ: FXR is a molecular target for the effects of vertical sleeve gastrectomy. Nature 509: 183-188, 2014.

7. Duran-Sandoval D, Cariou B, Percevault F, Hennuyer N, Grefhorst A, van Dijk TH, Gonzalez FJ, Fruchart JC, Kuipers F and Staels B: The farnesoid $\mathrm{X}$ receptor modulates hepatic carbohydrate metabolism during the fasting-refeeding transition. J Biol Chem 280: 29971-29979, 2005.

8. Zhang Y, Lee FY, Barrera G, Lee H, Vales C, Gonzalez FJ, Willson TM and Edwards PA: Activation of the nuclear receptor FXR improves hyperglycemia and hyperlipidemia in diabetic mice. Proc Natl Acad Sci USA 103: 1006-1011, 2006.
9. Cariou B, van Harmelen K, Duran-Sandoval D, van Dijk TH, Grefhorst A, Abdelkarim M, Caron S, Torpier G, Fruchart JC, Gonzalez FJ, et al: The farnesoid X receptor modulates adiposity and peripheral insulin sensitivity in mice. J Biol Chem 281: 11039-11049, 2006.

10. Downes M, Verdecia MA, Roecker AJ, Hughes R, Hogenesch JB, Kast-Woelbern HR, Bowman ME, Ferrer JL, Anisfeld AM, Edwards PA, et al: A chemical, genetic, and structural analysis of the nuclear bile acid receptor FXR. Mol Cell 11: 1079-1092, 2003.

11. Fang S, Suh JM, Reilly SM, Yu E, Osborn O, Lackey D, Yoshihara E, Perino A, Jacinto S, Lukasheva Y, et al: Intestinal FXR agonism promotes adipose tissue browning and reduces obesity and insulin resistance. Nat Med 21: 159-165, 2015.

12. Kong B, Luyendyk JP, Tawfik O and Guo GL: Farnesoid X receptor deficiency induces nonalcoholic steatohepatitis in low-density lipoprotein receptor-knockout mice fed a high-fat diet. J Pharmacol Exp Ther 328: 116-122, 2009.

13. Livak KJ and Schmittgen TD: Analysis of relative gene expression data using real-time quantitative PCR and the 2(-Delta Delta C(T)) method. Methods 25: 402-408, 2001.

14. Das M, Das S, Lekli I and Das DK: Caveolin induces cardioprotection through epigenetic regulation. J Cell Mol Med 16: 888-895, 2012.

15. Kong Q, Zhang H, Zhao T, Zhang W, Yan M, Dong X and Li P: Tangshen formula attenuates hepatic steatosis by inhibiting hepatic lipogenesis and augmenting fatty acid oxidation in $\mathrm{db} / \mathrm{db}$ mice. Int J Mol Med 38: 1715-1726, 2016.

16. de Oliveira PR, da Costa CA, de Bem GF, de Cavalho LC, de Souza MA, de Lemos Neto M, da Cunha Sousa PJ, de Moura RS and Resende AC: Effects of an extract obtained from fruits of Euterpe oleracea Mart. in the components of metabolic syndrome induced in C57BL/6J mice fed a high-fat diet. J Cardiovasc Pharmacol 56: 619-626, 2010.

17. Bogdanov P, Corraliza L, Villena JA, Carvalho AR, Garcia-Arumí J, Ramos D, Ruberte J, Simó R and Hernández C: The $\mathrm{db} / \mathrm{db}$ mouse: A useful model for the study of diabetic retinal neurodegeneration. PLoS One 9: e97302, 2014.

18. Duran-Sandoval D, Mautino G, Martin G, Percevault F, Barbier O, Fruchart JC, Kuipers F and Staels B: Glucose regulates the expression of the farnesoid $\mathrm{X}$ receptor in liver. Diabetes 53: 890-898, 2004.

19. Han CY, Kim TH, Koo JH and Kim SG: Farnesoid X receptor as a regulator of fuel consumption and mitochondrial function. Arch Pharm Res 39: 1062-1074, 2016.

20. Lambert G, Amar MJ, Guo G, Brewer HB Jr, Gonzalez FJ and Sinal CJ: The farnesoid X-receptor is an essential regulator of cholesterol homeostasis. J Biol Chem 278: 2563-2570, 2003.

21. Schonewille M, de Boer JF and Groen AK: Bile salts in control of lipid metabolism. Curr Opin Lipidol 27: 295-301, 2016.

22. Ronnett GV, Kleman AM, Kim EK, Landree LE and Tu Y: Fatty acid metabolism, the central nervous system, and feeding. Obesity (Silver Spring) 14 (Suppl 5): S201-S207, 2006.

23. Hardie DG and Pan DA: Regulation of fatty acid synthesis and oxidation by the AMP-activated protein kinase. Biochem Soc Trans 30: 1064-1070, 2002.

24. Carling D, Zammit VA and Hardie DG: A common bicyclic protein kinase cascade inactivates the regulatory enzymes of fatty acid and cholesterol biosynthesis. FEBS Lett 223: 217-222, 1987.

25. Ruderman NB, Saha AK and Kraegen EW: Minireview: Malonyl CoA, AMP-activated protein kinase, and adiposity. Endocrinology 144: 5166-5171, 2003.

26. Sierra AY, Gratacós E, Carrasco P, Clotet J, Ureña J, Serra D, Asins G, Hegardt FG and Casals N: CPT1c is localized in endoplasmic reticulum of neurons and has carnitine palmitoyltransferase activity. J Biol Chem 283: 6878-6885, 2008.

27. Noh K, Kim YM, Kim YW and Kim SG: Farnesoid X receptor activation by chenodeoxycholic acid induces detoxifying enzymes through AMP-activated protein kinase and extracellular signal-regulated kinase 1/2-mediated phosphorylation of CCAAT/enhancer binding protein $\beta$. Drug Metab Dispos 39: 1451-1459, 2011.

28. Hawley SA, Davison M, Woods A, Davies SP, Beri RK, Carling D and Hardie DG: Characterization of the AMP-activated protein kinase kinase from rat liver and identification of threonine 172 as the major site at which it phosphorylates AMP-activated protein kinase. J Biol Chem 271: 27879-27887, 1996. 
29. Gardès C, Chaput E, Staempfli A, Blum D, Richter H and Benson GM: Differential regulation of bile acid and cholesterol metabolism by the farnesoid X receptor in Ldlr -/- mice versus hamsters. J Lipid Res 54: 1283-1299, 2013.

30. Holloszy JO: Regulation by exercise of skeletal muscle content of mitochondria and GLUT4. J Physiol Pharmacol 59 (Suppl 7): S5-S18, 2008.

31. Aharoni-Simon M, Hann-Obercyger M, Pen S, Madar Z and Tirosh O: Fatty liver is associated with impaired activity of PPAR $\gamma$-coactivator $1 \alpha(\mathrm{PGC} 1 \alpha)$ and mitochondrial biogenesis in mice. Lab Invest 91: 1018-1028, 2011.

32. Morris EM, Meers GM, Booth FW, Fritsche KL, Hardin CD, Thyfault JP and Ibdah JA: PGC-1 $\alpha$ overexpression results in increased hepatic fatty acid oxidation with reduced triacylglycerol accumulation and secretion. Am J Physiol Gastrointest Liver Physiol 303: G979-G992, 2012.
33. Watanabe M, Houten SM, Wang L, Moschetta A, Mangelsdorf DJ, Heyman RA, Moore DD and Auwerx J: Bile acids lower triglyceride levels via a pathway involving FXR, SHP, and SREBP-1c. J Clin Invest 113: 1408-1418, 2004.

34. Zhang Y, Castellani LW, Sinal CJ, Gonzalez FJ and Edwards PA: Peroxisome proliferator-activated receptor-gamma coactivator 1alpha (PGC-1alpha) regulates triglyceride metabolism by activation of the nuclear receptor FXR. Genes Dev 18: 157-169, 2004

35. Matsukuma KE, Bennett MK, Huang J, Wang L, Gil G and Osborne TF: Coordinated control of bile acids and lipogenesis through FXR-dependent regulation of fatty acid synthase. J Lipid Res 47: 2754-2761, 2006. 